\title{
Accommodating Changes in Semistructured Databases Using Multidimensional OEM
}

\author{
Yannis Stavrakas ${ }^{1,2}$, Manolis Gergatsoulis $^{2}$, Christos Doulkeridis $^{1}$, and \\ Vassilis Zafeiris ${ }^{1}$ \\ 1 Knowledge \& Database Systems Laboratory \\ National Technical University of Athens (NTUA), 15773 Athens, Greece. \\ ${ }^{2}$ Institute of Informatics \& Telecommunications, \\ National Centre for Scientific Research (N.C.S.R.) 'Demokritos', \\ 15310 Aghia Paraskevi Attikis, Greece. \\ \{cdoulk, bzafiris\}@aueb.gr
}

\begin{abstract}
Multidimensional Semistructured Data (MSSD) are semistructured data that present different facets under different contexts. Contexts represent alternative worlds, and are expressed by assigning values to a set of user-defined variables called dimensions. The notion of context has been incorporated in OEM, and the extended model is called Multidimensional OEM (MOEM), a graph model for MSSD. In this paper, we explain in detail how MOEM can represent the history of an OEM database. We discuss how MOEM properties are applied in the case of representing OEM histories, and show that temporal OEM snapshots can be obtained from MOEM. We present a system that implements the proposed ideas, and we use an example scenario to demonstrate how an underlying MOEM database accommodates changes in an OEM database. Furthermore, we show that MOEM is capable to model changes occurring not only in OEM databases, but in Multidimensional OEM databases as well.
\end{abstract}

Keywords: Multidimensional Semistructured Data, Multidimensional OEM, OEM History, History of Semistructured Data.

\section{Introduction and Preliminaries}

In this paper we investigate the use of Multidimensional Object Exchange Model (Multidimensional OEM or MOEM for short), a graph model for multidimensional semistructured data, for representing histories of semistructured databases. We start with an introduction to Multidimensional OEM, we explain in detail the way it can be used to model the history of an OEM database, we present an example scenario using our prototype implementation, and we show that Multidimensional OEM can be used to model its own histories as well.

The motivation behind multidimensional semistructured data is that, in contrast to traditional databases and information systems where the number of users is more or less known and their background is to a great extend homogeneous, Web users do not share the same background and do not apply the same conventions when interpreting data. Such users can have different perspectives of the 
same entities, a situation that should be taken into account by Web data models. As a simple example, imagine a report that must be represented in various languages and at various degrees of detail.

In this section we review some preliminary concepts on multidimensional semistructured data, that will be used in the sections that follow. Multidimensional semistructured data (MSSD in short) [9] are semistructured data [10,1] which present different facets under different contexts. The main difference between conventional and multidimensional semistructured data is the introduction of context specifiers. Context specifiers are syntactic constructs that are used to qualify semistructured data expressions (ssd-expressions) [1] and specify sets of worlds under which the corresponding ssd-expressions hold. In this way, it is possible to have at the same time variants of the same information entity, each holding under a different set of worlds. An information entity that encompasses a number of variants is called multidimensional entity, and its variants are called facets of the entity. The facets of a multidimentional entity may differ in value and / or structure, and can in turn be multidimensional entities or conventional information entities. Each facet is associated with a context that defines the conditions under which the facet becomes a holding facet of the multidimensional entity.

In [9] we extend ssd-expressions [1] with context specifiers and propose $m s s d-$ expressions, a syntax for representing multidimensional semistructured data. Another way of representing multidimensional semistructured data is Multidimensional XML (MXML in short) [5,6], an extension of XML that incorporates context specifiers. In MXML, multidimensional elements and multidimensional attributes may have different facets that depend on a number of dimensions. MXML gives new possibilities for designing Web pages that deal with context-dependent data. We refer to the new method as the multidimensional paradigm, and we present it in detail in [6].

\subsection{Context and Dimensions}

The notion of world is fundamental in MSSD. A world represents an environment under which data obtain a substance. In the following definition, we specify the notion of world using a set of parameters called dimensions.

Definition 1. Let $\mathcal{D}$ be a nonempty set of dimension names and for each $d \in \mathcal{D}$, let $\mathcal{V}_{d}$ be the domain of $d$, with $\mathcal{V}_{d} \neq \emptyset$. A world $w$ with respect to $\mathcal{D}$ is a set whose elements are pairs $(d, v)$, where $d \in \mathcal{D}$ and $v \in \mathcal{V}_{d}$, such that for every dimension name in $\mathcal{D}$ there is exactly one element in $w$.

In MSSD, sets of worlds are represented by context specifiers, which can be seen as constraints on dimension values.

Example 1. The use of dimensions for representing worlds is shown with the following three context specifiers:

(a) $[$ time $=07: 45]$

(b) [language=greek, detail in \{low,medium\}]

(c) [season in $\{$ fall, spring $\}$, daytime=noon $\mid$ season=summer] 
In Example 1, context specifier (a) represents the worlds for which the dimension time has the value $07: 45$, while (b) represents the worlds for which language is greek and detail is either low or medium. Context specifier (c) is more complex, and represents the worlds where season is either fall or spring and daytime is noon, together with the worlds where season is summer. Notice that, according to definition 1, for a set of (dimension, value) pairs to represent a world with respect to a set of dimensions $\mathcal{D}$, it must contain exactly one pair for each dimension in $\mathcal{D}$. Therefore, if $\mathcal{D}=\{$ language, detail $\}$ with $\mathcal{V}_{\text {language }}=\{$ english, greek $\}$ and $\mathcal{V}_{\text {detail }}=\{$ low, medium, high $\}$, then $\{($ language, greek $),($ detail, low $)\}$ is one of the six possible worlds with respect to $\mathcal{D}$. This world is represented by context specifier (b) in Example 1, together with the world \{(language, greek), (detail,medium) $\}$. It is not necessary for a context specifier to contain values for every dimension in $\mathcal{D}$. Omitting a dimension implies that its value may range over the whole dimension domain.

The context specifier [] is called universal context and represents the set of all possible worlds with respect to any set of dimensions $\mathcal{D}$. In [9] we have defined operations on context specifiers, such as context intersection and context union that correspond to the conventional set operations of intersection and union on the related sets of worlds. We have also defined how a context specifier can be transformed to the set of worlds it represents with respect to a set of dimensions $\mathcal{D}$. An important case for MSSD is when two context specifiers represent disjoint sets of worlds; in that case the context specifiers are called mutually exclusive.

\subsection{Multidimensional OEM}

Multidimensional Object Exchange Model (MOEM) is an extension of Object Exchange Model (OEM) [2], suitable for representing multidimensional semistructured data. MOEM extends OEM with two new basic elements:

- Multidimensional nodes: represent multidimensional entities, and are used to group together nodes that constitute facets of the entities, playing the role of surrogates for these facets. Graphically, multidimensional nodes have a rectangular shape to distinguish them from conventional circular nodes.

- Context edges: are directed labeled edges that connect multidimensional nodes to their variants. The label of a context edge pointing to a variant $p$, is a context specifier defining the set of worlds under which $p$ holds. Context edges are drawn as thick lines, to distinguish them from conventional (thin-lined) OEM edges called entity edges in MOEM.

In MOEM the conventional circular nodes of OEM are called context nodes and they represent facets associated with some context. Conventional OEM edges (thin-lined) are called entity edges and define relationships between objects. As in OEM, all MOEM nodes are considered objects, and have a unique object identifier (oid). Context objects are divided into complex objects and atomic objects. Atomic objects have a value from one of the basic types, e.g. integer, real, strings, etc. A context edge cannot start from a context node, and an entity edge cannot start from a multidimensional node. Those two are the only constraints on the morphology of an MOEM graph. 


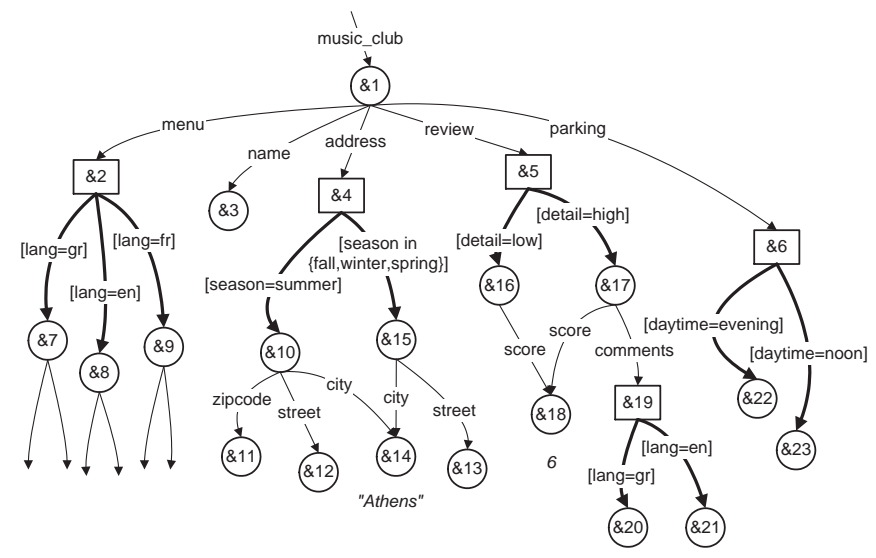

Fig. 1. A multidimensional music-club.

As an example, consider the MOEM graph in Figure 1 which represents context-dependent information about a music-club. The graph is not fully developed and some of the atomic objects do not have values attached. The music_club with oid \& 1 operates on a different address during the summer than the rest of the year (in Athens it is not unusual for clubs to move south close to the sea in the summer period, and north towards the city center during the rest of the year). Except from having a different value, context objects can have a different structure, as is the case of \&10 and \&15 which are facets of the multidimensional object address with oid \&4. The menu of the club is available in three languages, namely English, French and Greek. In addition, the club has a couple of alternative parking places, depending on the time of day as expressed by the dimension dayt ime. The music-club review has two facets: node $\& 16$ is the low detail facet containing only the review score with value 6 , while the high detail facet $\& 17$ contains in addition review comments in two languages.

The notion of multidimensional data graph is formally defined as follows.

Definition 2. Let $\mathcal{C}$ be a set of context specifiers, $\mathcal{L}$ be a set of labels, and $\mathcal{A}$ be a set of atomic values. A multidimensional data graph is a finite directed edge-labeled multigraph $G=(V, E, r, \mathcal{C}, \mathcal{L}, \mathcal{A}, v)$, where: (1) The set of nodes $V$ is partitioned into multidimensional nodes and context nodes $V=V_{m l d} \cup V_{c x t}$. Context nodes are further divided into complex nodes and atomic nodes $V_{c x t}=$ $V_{c} \cup V_{a}$. (2) The set of edges $E$ is partitioned into context edges and entity edges $E=E_{c x t} \cup E_{\text {ett }}$, such that $E_{c x t} \subseteq V_{\text {mld }} \times \mathcal{C} \times V$ and $E_{\text {ett }} \subseteq V_{c} \times \mathcal{L} \times V$. (3) $r \in V$ is the root, with the property that there exists a path from $r$ to every other node in $V$. (4) $v$ is a function that assigns values to nodes, such that: $v(x)=M$ if $x \in V_{m l d}, v(x)=C$ if $x \in V_{c}$, and $v(x)=v^{\prime}(x)$ if $x \in V_{a}$, where $M$ and $C$ are reserved values, and $v^{\prime}$ is a value function $v^{\prime}: V_{a} \rightarrow \mathcal{A}$ which assigns values to atomic nodes.

An MOEM graph is a context deterministic multidimensional data graph, that is, a multidimensional data graph whose context edges that depart from the same 
multidimensional node have labels (context specifiers) that are mutually exclusive. Two fundamental concepts related to multidimensional data graphs and MOEMs are the notions of explicit and inherited context [9]. The explicit context of a context edge is the context specifier assigned to that edge, while the explicit context of an entity edge is considered to be the universal context specifier []. The explicit context can be considered as the "true" context only within the boundaries of a single multidimensional entity. When entities are connected together in a MOEM graph, the explicit context of an edge is not the "true" context, in the sense that it does not alone determine the worlds under which the destination node holds. The reason for this is that, when an entity $e_{2}$ is part of (pointed by through an edge) another entity $e_{1}$, then $e_{2}$ can have substance only under the worlds that $e_{1}$ has substance. This can be conceived as if the context under which $e_{1}$ holds is inherited to $e_{2}$. The context propagated in that way is combined with (constraint by) the explicit context of each edge to give the inherited context for that edge. In contrast to edges, nodes do not have an explicit context; like edges, however, they do have an inherited context. The inherited context of a node or edge gives the set of worlds under which the node or edge is taken into account, when reducing the MOEM graph to a conventional OEM graph (as explained later in this section).

Multidimensional entities are not obliged to have a facet under every possible world. However, they must provide enough coverage to give substance to each incoming edge under at least one world. The notion of validity [9] of an MOEM graph ensures that edges pointing to multidimensional nodes do not exist in vain. In particular, an edge $h$ leading to a node $q$ is invalid if the inherited context of $h$ has no common world with the union of the worlds represented by the explicit contexts of the edges that depart from $q$.

Given a specific world, it is always possible to reduce a context-deterministic multidimensional data graph (or MOEM) to a conventional OEM graph holding under that world. A procedure that performs this MOEM reduction is presented in [9]. In addition, given a set of worlds, it is possible to partially reduce an MOEM into a new MOEM, that encompasses only the OEM facets for the given set of worlds.

\section{Representing Histories of Semistructured Data with MOEM}

In this section, we show how Multidimensional OEM can be used to represent changes in an OEM database. The problem can be stated as follows: given a static OEM graph that comprises the database, we would like a way to represent dynamically changes in the database as they occur, keeping a history of transitions, so that we are able to subsequently query on those changes. In [9] we outlined some preliminary ideas towards a method for modeling OEM histories, and showed that it is feasible to model such histories through MOEM. In this paper we further extend those ideas and present the method in detail: we give specific algorithms, discuss how MOEM properties are applied, present the $O E M$ History application and give a complete example scenario. In addition, we show that MOEM is expressive enough to represent its own histories as well. 
The problem of representing and querying changes in semistructured data has also been studied in [4], where Delta OEM (DOEM in short) has been proposed. DOEM is a graph model that extends OEM with annotations containing temporal information. Four basic change operations, namely creNode, updNode, addArc, and remArc are considered by the authors in order to modify an OEM graph. Those operations are mapped to four types of annotations. Annotations are tags attached to a node or an arc, containing information that encodes the history of changes for that node or arc. When an OEM basic change operation takes place, a new annotation is added to the affected node or arc, stating the type of the operation, the timestamp, and in the case of updNode the old value of the object. The modifications suggested by the basic change operations actually take place, except from the arc removal which results to just annotating the arc. Our approach is based on the same framework, and builds on the key concepts presented in [4]. It is however quite different, as changes are represented by introducing new facets instead of adding annotations.

A special graph for modeling the dynamic aspects of semistructured data, called semistructured temporal graph is proposed in [8]. In this graph, every node and edge has a label that includes a part stating the valid interval for the node or edge. Modifications in the graph cause changes in the temporal part of labels of affected nodes and edges.

An approach for representing temporal XML documents is proposed in [3], where leaf data nodes can have alternative values, each holding under a time period. However, the model presented in [3] does not allow dimensions other than time, and does not explicitly support facets with varying structure for nodes that are not leaves. Another approach for representing time in XML documents is described in [7], where the use of Multidimensional XML is suggested.

An important advantage of MOEM over those approaches is that a single model can be applied to a variety of problems from different fields; representing valid time is just one of the possible applications of MOEM. MOEM is suitable for modeling entities that present different facets, a problem often encountered on the Web, and the representation of semistructured database histories can be seen as a special case of this problem. Properties and processes defined for the general case of MOEM, like inherited context, validity, reduction, and querying are also used without change in the case of representing semistructured histories. In addition, as we show in section 4, MOEM is a model capable of representing its own histories.

\subsection{OEM and MOEM Basic Operations}

A conventional OEM graph is defined in [2] as a quadruple $O=(V, E, r, v)$, where $V$ is a set of nodes, $E$ a set of labeled directed edges $(p, l, q)$ where $p, q \in V$ and $l$ is a string, $r$ is a special node called the root, and $v$ is a function mapping each node to an atomic value of some type (int, string, etc.), or to the reserved value $C$ which denotes a complex object. In order to modify an OEM database $O$, four basic change operations were identified in [4]:

$\operatorname{creNode}(\boldsymbol{n i d}, \boldsymbol{v a l})$ : creates a new node, where nid is a new node oid (nid $\notin$ $V$ ), and $v a l$ is an atomic value or the reserved value $C$. 
updNode $($ nid, $\boldsymbol{v a l})$ : changes the value of an existing object nid to a new value val. The node nid must not have any outgoing arcs (in case its old value is $C$, the arcs should have been removed prior to updating the value).

$\operatorname{addArc}(p, l, q)$ : adds a new arc labeled $l$ from object $p$ to object $q$. Both nodes $p$ and $q$ must already exist in $V$, and $(p, l, q)$ must not exist in $E$.

$\operatorname{remArc}(p, l, q)$ : removes the existing arc $(p, l, q)$. Both nodes $p$ and $q$ must exist in $V$.

Given an MOEM database $M=(V, E, r, \mathcal{C}, \mathcal{L}, \mathcal{A}, v)$, we introduce the following basic operations for changing $M$.

createCNode $(\boldsymbol{c i d}, \boldsymbol{v a l})$ : a new context node is created. The identifier $c i d$ is new and must not occur in $V_{c x t}$. The value val can be an atomic value of some type, or the reserved value $C$.

updateCNode $(\boldsymbol{c i d}, \boldsymbol{v a l})$ : changes the value of $c i d \in V_{c x t}$ to val. The node must not have any outgoing arcs.

createMNode $(\mathbf{m i d})$ : a new multidimensional node is created. The identifier mid is new and must not occur in $V_{m l d}$.

addEEdge $(\boldsymbol{c i d}, \boldsymbol{l}, \boldsymbol{i d})$ : creates a new entity edge with label $l$ from node $c i d$ to node $i d$, where $c i d \in V_{c x t}$ and $i d \in V$.

remEEdge $(\boldsymbol{c i d}, \boldsymbol{l}, \boldsymbol{i d})$ : removes the entity edge $(c i d, l, i d)$ from $M$. The edge $(c i d, l, i d)$ must exist in $E_{\text {ett }}$.

addCEdge $($ mid, context, $\boldsymbol{i d})$ : creates a new context edge with context context from node mid to node $i d$, where mid $\in V_{\text {mld }}$ and $i d \in V$.

remCEdge (mid, context, $i d)$ : removes the context edge (mid,context,id) from $M$. The context edge (mid, context, $i d$ ) must exist in $E_{c x t}$.

For conventional OEM and MOEM, object deletion is achieved through arc removal, since in both OEM and MOEM the persistence of an object is determined by whether or not the object is reachable from the root. Sometimes the result of a single basic operation $u$ leads to an inconsistent state: for instance, when a new object is created, it is temporarily unreachable from the root. In practice however, it is typical to have a sequence $L=u_{1}, u_{2}, \ldots, u_{n}$ of basic operations $u_{i}$, which corresponds to a higher level modification to the database. By associating such higher level modifications with a timestamp, an OEM history $H$ is defined as a sequence of pairs $(t, U)$, where $U$ denotes a set of basic change operations that corresponds to $L$ as defined in [4], and $t$ is the associated timestamp. Note that within a single sequence $L$, a newly created node may be unreachable from the root and still not be considered deleted. At the end of each sequence, however, unreachable nodes are considered deleted and cannot be referenced by subsequent operations.

\subsection{Using MOEM to Model OEM Histories}

We will now use the operations defined in the previous section to represent changes in an OEM database using MOEM. Our approach is to map the four OEM basic change operations to MOEM basic operations, in such a way, that new facets of an object are created whenever changes occur in that object. In this manner, the initial OEM database $O$ is transformed into an MOEM graph, that uses a dimension $d$ whose domain is time to represent an OEM history $H$ valid [4] for $O$. 
We assume that our time domain $T$ is linear and discrete; we also assume: (1) a reserved value now, such that $t<$ now for every $t \in T$, (2) a reserved value start, representing the start of time, and (3) a syntactic shorthand $v_{1} . . v_{n}$ for discrete and totally ordered domains, meaning all values $v_{i}$ such that $v_{1} \leq v_{i} \leq v_{n}$. The time period during which a context node is the holding node of the corresponding multidimensional entity is denoted by qualifying that context node with a context specifier of the form $\left[\mathrm{d}\right.$ in $\left.\left\{t_{1} \ldots t_{2}\right\}\right]$.

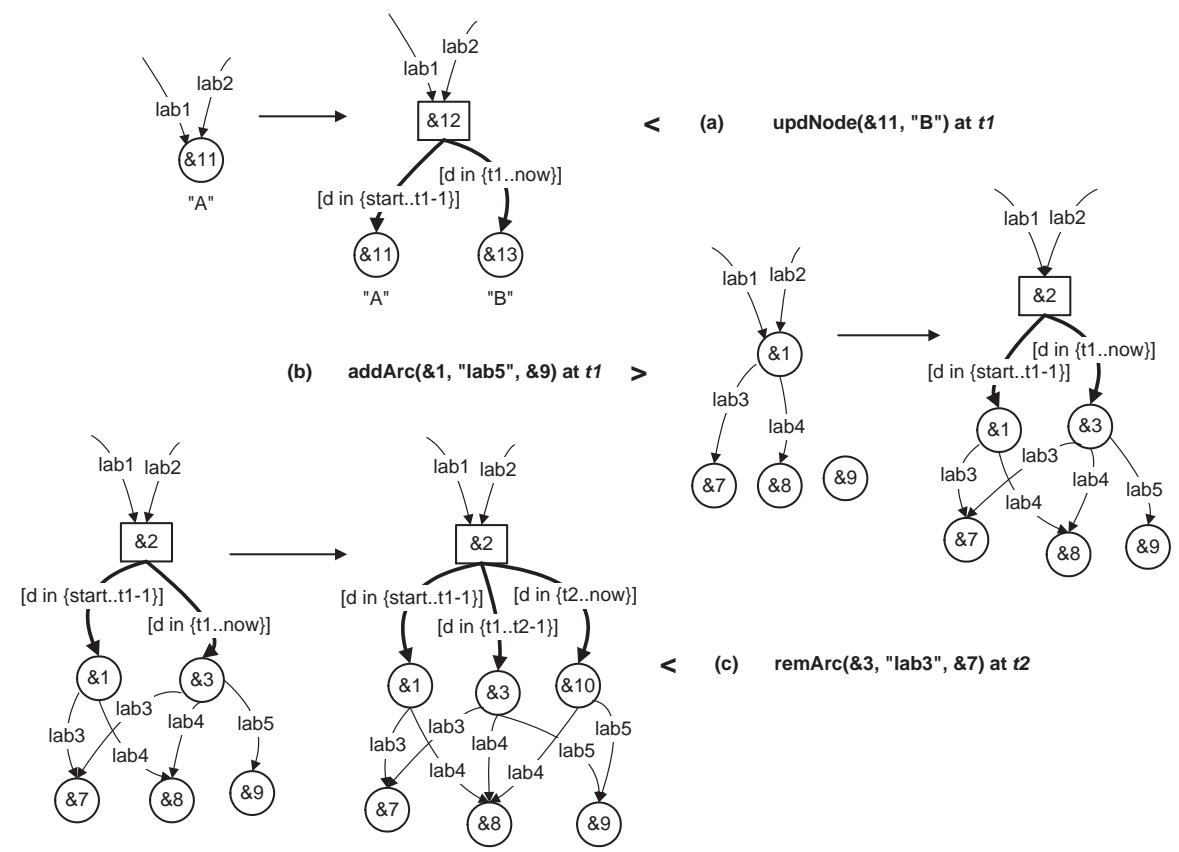

Fig. 2. Modeling OEM basic change operations with MOEM.

Figure 2 gives an intuition about the correspondence between OEM and MOEM operations. Consider the sets $U_{1}$ and $U_{2}$ of basic change operations, with timestamps $t_{1}$ and $t_{2}$ respectively. Figure 2(a) shows the MOEM representation of an atomic object, whose value " $\mathrm{A}$ " is changed to " $\mathrm{B}$ " through a call to the basic change operation updNode of $U_{1}$. Figure 2(b) shows the result of addArc operation of $U_{1}$, while figure 2(c) shows the result of remArc operation of $U_{2}$, on the same multidimensional entity. It is interesting to notice that three of the four OEM basic change operations are similar, in that they update an object be it atomic (updNode) or complex (addArc, remArc), and all three are mapped to MOEM operations that actually update a new facet of the original object. Creating a new node with creNode does not result in any additional MOEM operations; the new node will subsequently be linked with the rest of the graph (within the same set $U$ ) through addArc operation(s), which will cause new object facet(s) to be created. Note that, although object identifiers in Figure 2 may change during the 
OEM history, this is more an implementation issue and does not present any real problem. In addition, it is worth noting that the changes induced by the OEM basic change operations affect only localized parts of the MOEM graph, and do not propagate throughout the graph.

Having outlined the approach, we now give a detailed specification. First, the following four utility functions and procedures are defined.

id1 $\leftarrow \mathbf{m d}(\mathbf{i d 2})$, with $i d 1, i d 2 \in V$. Returns the multidimensional node for a context node, if it exists. If $i d 2 \in V_{c x t}$ and there exists an element (mid,context, $i d$ ) in $E_{c x t}$ such that $i d=i d 2$, then mid is returned. If $i d 2 \in V_{c x t}$ and no corresponding context edge exists, $i d 2$ is returned. If $i d 2 \in V_{m l d}$, $i d 2$ is returned. Notice that there is at most one multidimensional node pointing to any context node, in other words for every cid $\in V_{c x t}$ there is at most one mid such that ( mid, context, cid $) \in E_{\text {cxt }}$. However, this is a property of MOEM for the specific problem, because of the special way the MOEM graph is constructed for representing histories, and is not the general case.

boolean $\leftarrow$ withinSet(cid), with cid $\in V_{c x t}$. Checks whether the context node cid is created within the current set $U$ of basic change operations. This function is used while change operations are in progress, and returns true if cid was created within the same set. It returns false if cid was created within a previous set of operations.

The following procedure mEntity(id), with $i d \in V_{c x t}$, creates a new multidimensional node mid pointing to $i d$, and redirects all incoming edges from $i d$ to mid. Note that the procedure alters the graph, but not the information modeled by the graph: the multidimensional entity created by the procedure has $i d$ as its only facet holding under every world.

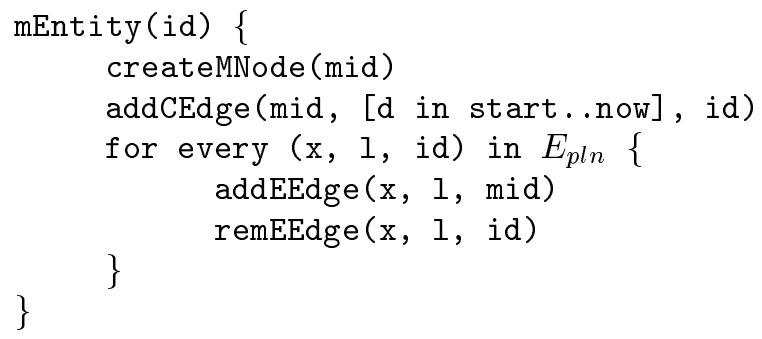

In the procedure newCxt(id1, id2, ts), with $i d 1, i d 2 \in V_{c x t}$ and $t s \in T$, $i d 1$ is the currently most recent facet of a multidimensional entity, and $i d 2$ is a new facet that is to become the most recent. The procedure arranges the context specifiers accordingly.

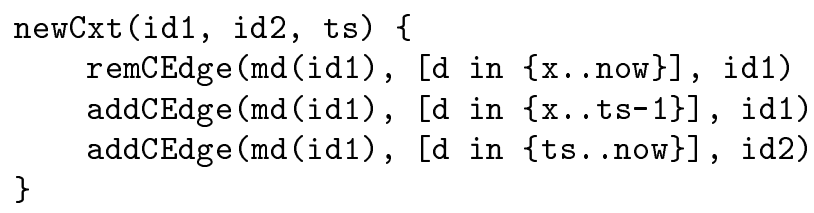

The next step is to show how each OEM basic change operation is implemented using the basic MOEM operations. We assume that each of the OEM operations 
is part of a set $U$ with timestamp ts, and that the node $p$ is the most recent context node of the corresponding multidimensional entity, if such an entity exists. This is because changes always happen to the current snapshot of OEM, which corresponds to the most recent facets of MOEM multidimensional entities. The most recent context node is the one holding in current time, i.e. the node whose context specifier is of the form [d in \{somevalue. now $\}]$.

$\operatorname{updNode}(p, \boldsymbol{n e w v a l})$ : If $p$ has been created within $U$, its value is updated directly, and the process terminates. Otherwise, if $p$ is not pointed to by a multidimensional node, a new multidimensional node is created for $p$, having $p$ as its only context node with context specifier [d in \{start..now $\}]$. A new facet is then created with value newval, and becomes the most recent facet by adjusting the relevant context specifiers. Since a node updated by updNode cannot have outgoing edges, no edge copying takes place in contrast to the case of addArc that follows.

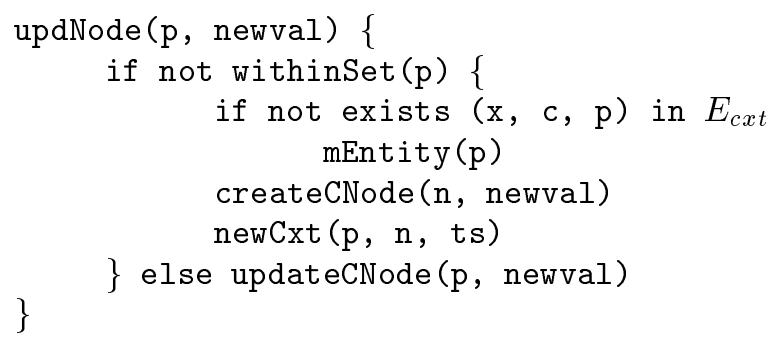

$\operatorname{addArc}(p, \boldsymbol{l}, \boldsymbol{q})$ : If $p$ has been created within $U$, it is used directly: the new arc is added, and the process terminates. Otherwise, if $p$ is not already pointed to by a multidimensional node, a new multidimensional node is created for $p$, having $p$ as its only context node with context specifier [d in \{start..now $\}]$. A new "clone" facet $n$ is then created by copying all outgoing edges of $p$ to $n$. In this case, the context specifiers are adjusted so that $t s$ is taken into account, and $n$ becomes the most recent facet as depicted in figure 2(b) for $t s=t 1$. Finally the new edge specified by the basic change operation is added to the most recent facet. Note that, in the frame of representing changes, an MOEM is constructed in such a way that an entity edge does not point directly to a context node $q_{c}$ if there exists a context edge $\left(q_{m}, c, q_{c}\right)$; instead, it always points to the corresponding multidimensional node $q_{m}$, if $q_{m}$ exists. This is achieved by using the function $\operatorname{md}(q)$ in combination with $m \operatorname{Entity}(p)$.

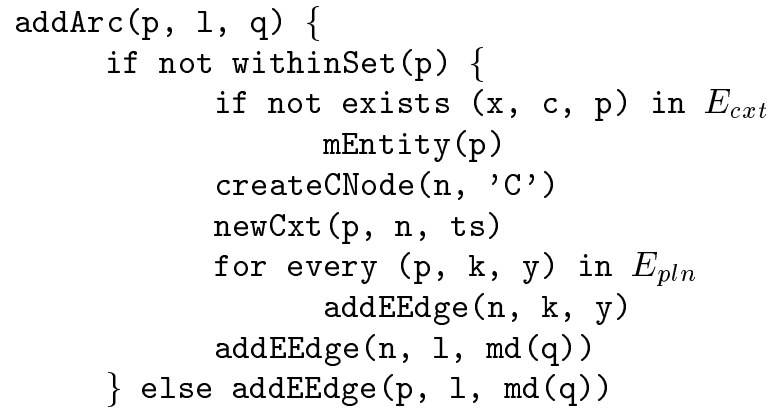


$\operatorname{remArc}(p, l, q):$ The process is essentially the same as $\operatorname{add} \operatorname{Arc}(p, l, q)$, with the difference of removing an edge at the end of the process, instead of adding one. Therefore, remArc is like addArc, except for the last two calls to addEEdge which are replaced with calls to remEEdge with the same arguments.

$\operatorname{creNode}(\boldsymbol{p}, \boldsymbol{v} \boldsymbol{a l})$ : this basic change operation is mapped to createCNode( $p$, val) with no further steps. New facets will be created when new edges are added to connect node $p$ to the rest of the graph.

\subsection{Applying MOEM Properties}

MOEM graphs that represent OEM histories have special characteristics, not generally encountered in MOEM graphs. In this section we discuss how those characteristics affect the MOEM properties of inherited context, validity, and reduction to conventional OEMs.

Let $G$ be a multidimensional data graph produced by the process specified in section 2.2 , let $e$ be a multidimensional entity in $G$, with multidimensional node $m$ and facets $e_{1}, e_{2}, \ldots, e_{n}$, and let $c_{1}, c_{2}, \ldots, c_{n}$ be the context specifiers of the respective context edges. Notice that, as already stated, the process in section 2.2 guarantees that at most one multidimensional node points to any context node. In addition, in the case of representing an OEM history, worlds are time instances. It is easy to observe that $G$ is context deterministic, because for every multidimensional entity $e$ in $G$, the contexts $c_{1}, c_{2}, \ldots, c_{n}$ always define disjoint sets of worlds, thus for any given time instance at most one of $e_{1}, e_{2}, \ldots, e_{n}$ may hold. Consequently, $G$ is an MOEM graph, and the reduction process (defined in [9]) will always give an OEM graph, for any time instance in $T$.

In addition, from the procedures mEntity and newCxt defined in section 2.2, it can be seen that: (a) $c_{1}$ has the form [d in $\left\{\right.$ start.. somevalue1\}], (b) $c_{n}$ has the form [d in \{somevalueN. .now\}], and (c) the union of the context specifiers $c_{1}, c_{2}, \ldots, c_{n}$ can be represented by [d in $\{$ start..now $\}$, for every $e$ in $G$. Recall that an edge pointing to $m$ is invalid if its inherited context represents worlds that are disjoint with every $c_{1}, c_{2}, \ldots, c_{n}$. This however, is not possible in our case since $\{$ start. .now covers the whole time domain. Consequently, every edge in $G$ is valid, therefore $G$ is valid. Edge validity ensures that there exists (at least) a world under which the edge "survives" (is part of the OEM holding under that world after reducing MOEM).

Although for every multidimensional entity $e$ in $G$ the corresponding context specifiers $c_{1}, c_{2}, \ldots, c_{n}$ cover the complete $\{$ start. .now time range, the corresponding inherited contexts denote the true life span of the entity and its facets. To understand why, note that each multidimensional entity $e$ in $G$ corresponds to a node that existed at some time in the evolution of the OEM graph. The facets of $e$ correspond to OEM changes that had affected that node. Edges pointing to $m$ correspond to edges that pointed to that node at some time in the evolution of the OEM graph. In addition, the inherited context of edges pointing to $m$ will be such as to allow to each one of $e_{1}, e_{2}, \ldots, e_{n}$ to "survive" under some world. Therefore, for every $e_{i}$ with $2 \leq i \leq n-1$ the explicit context $c_{i}$ is also the inherited context 
of the context node $e_{i}$. As we have seen, $c_{1}=[\mathrm{d}$ in $\{$ start. . somevalue 1$\}]$, and $c_{n}=$ [d in $\{$ somevalueN. .now $\left.\}\right]$; for facets $e_{1}$ and $e_{n}$ incoming edges restrict the explicit contexts, so that the inherited context of $e_{1}$ may have a first value greater than start, while the inherited context of $e_{n}$ may have a second value smaller than now.

It is now easy to understand the result of applying MOEM reduction to $G$. Given an OEM database $O$ and an MOEM database $G$ that represents the history of $O$ as defined above, it is possible to specify a time instance $t$ and reduce $G$ to an OEM database $O^{\prime}$. Then $O^{\prime}$ will be the snapshot of $O$ at the time instance $t$.

\section{OEM History}

OEM History is an application developed in Java, which implements the method described in Section 2 for representing OEM histories. As it can be seen in Figure 3, OEM History employs a multi-document interface (MDI) with each internal window displaying a data graph. There are two main windows : one that displays an MOEM graph that corresponds to the internal model of the application, and one that always shows the current state of the OEM database. Furthermore, the user can ask for a snapshot of the database for any time instance in $T$ (the time domain), that will be presented as an OEM graph in a separate window. The toolbar on the left side contains buttons that correspond to the four OEM basic change operations, which can be used only on the window with the OEM depicting the current state of the database. These operations are mapped to a number of operations that update the internal MOEM data model of the application, which is the only model actually maintained by OEM History. The current OEM database is the result of an MOEM reduction for $d=$ now.

Note that the "tick" button in the left toolbar removes nodes that are not accessible from the root. The last button in the toolbar marks the end of a sequence of basic change operations, and commits all changes to the database under a common timestamp. Operations like MOEM reduction and MOEM validity check can be initiated from the upper toolbar or from the application menu.

In Figure 3, we see the initial state of an OEM database containing information about the employees of a company, and the corresponding MOEM graph. The right window displays the underlying MOEM model, while the left window displays the result of the MOEM reduction for $d=$ now.

Figure 4 (a) shows the current state of the OEM database and the corresponding MOEM graph after a couple of change sequences. First, at the time instance 10 the salary of John has been increased from 1000 to 2000 . Then, at the time instance 20 a new employee called Peter joined the company with salary 3000 .

In Figure 4 (b) two more change sequences have been applied. The salary of Peter increased to 4000 at the time instance 30, and at the time instance 40 Peter left the company. Note that, as shown on the caption, the left window does not display the current OEM. Instead it depicts a snapshot of the OEM database for the time instance 5, which is obtained from reducing the MOEM in the right window for $d=5$. That snapshot is identical to the initial state of the database, since the first change occurred at the time instance 10. 


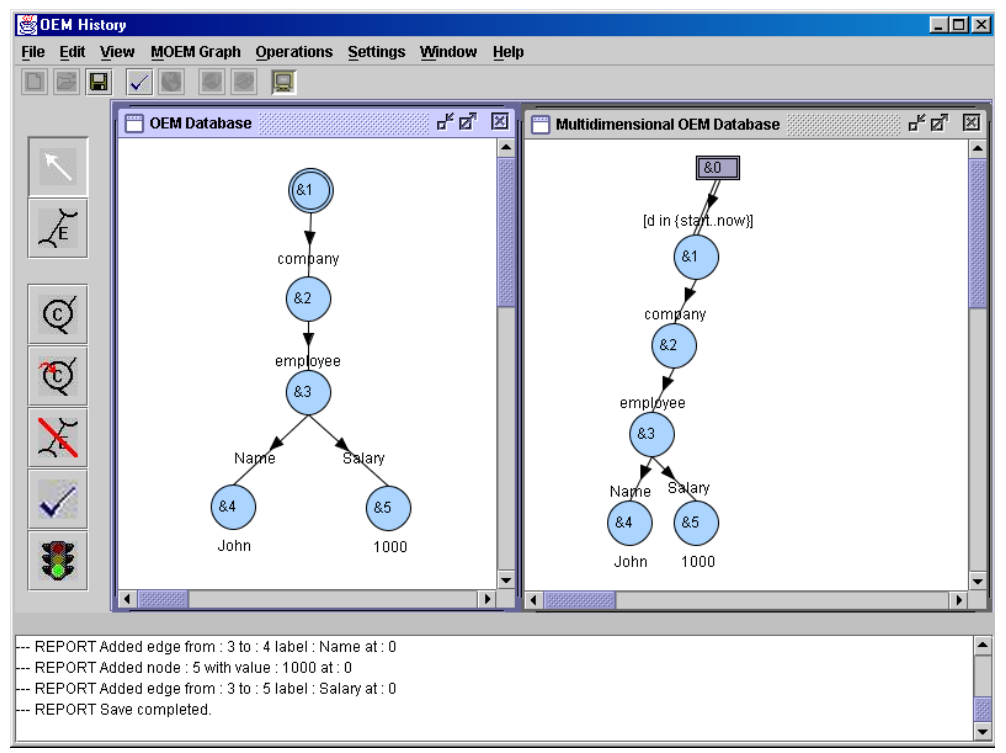

Fig. 3. Initial state of example database in OEM History application.

OEM History is available at:

http://www.dblab.ntua.gr/ ys/moem/moem.html

\section{Representing Changes in MOEM Databases}

Besides representing the history of OEM databases as shown in section 2, MOEM has another interesting property. In this section we show that MOEM is expressive enough to model its own histories. In other words, for any MOEM database $G$ evolving over time it is possible to have an MOEM database $G^{\prime}$, such that $G^{\prime}$ represents the history of $G$.

The approach is similar to that of section 2.2; we show that any of the MOEM basic operations (defined in section 2.1) applied to $G$, can be mapped to a number of MOEM basic operations on $G^{\prime}$, in such a way that $G^{\prime}$ represents the history of $G$. Figure 5 gives the intuition about this mapping, for three basic operations. Context edge labels $c 1, c 2, \ldots, c N$ are context specifiers involving any number of dimensions, as in the example of Figure 1, while the dimension $d$ is defined in section 2.2. Note that the use of dimension $d$ in $G^{\prime}$ does not preclude $G$ from using other dimensions that range over time domains. The MOEM operations depicted in Figure 5 are basic operations occurring on $G$, and the corresponding graphs show how those operations transform $G^{\prime}$. For simplicity, graphs on the left side do not contain context specifiers with the dimension $d$, and all timestamps are $t 1$. It is however easy to envisage the case where $d$ is also on the left side and timestamps progressively increase in value, if we look at Figure 2 (b) and (c) which follow a similar pattern. 
(a)
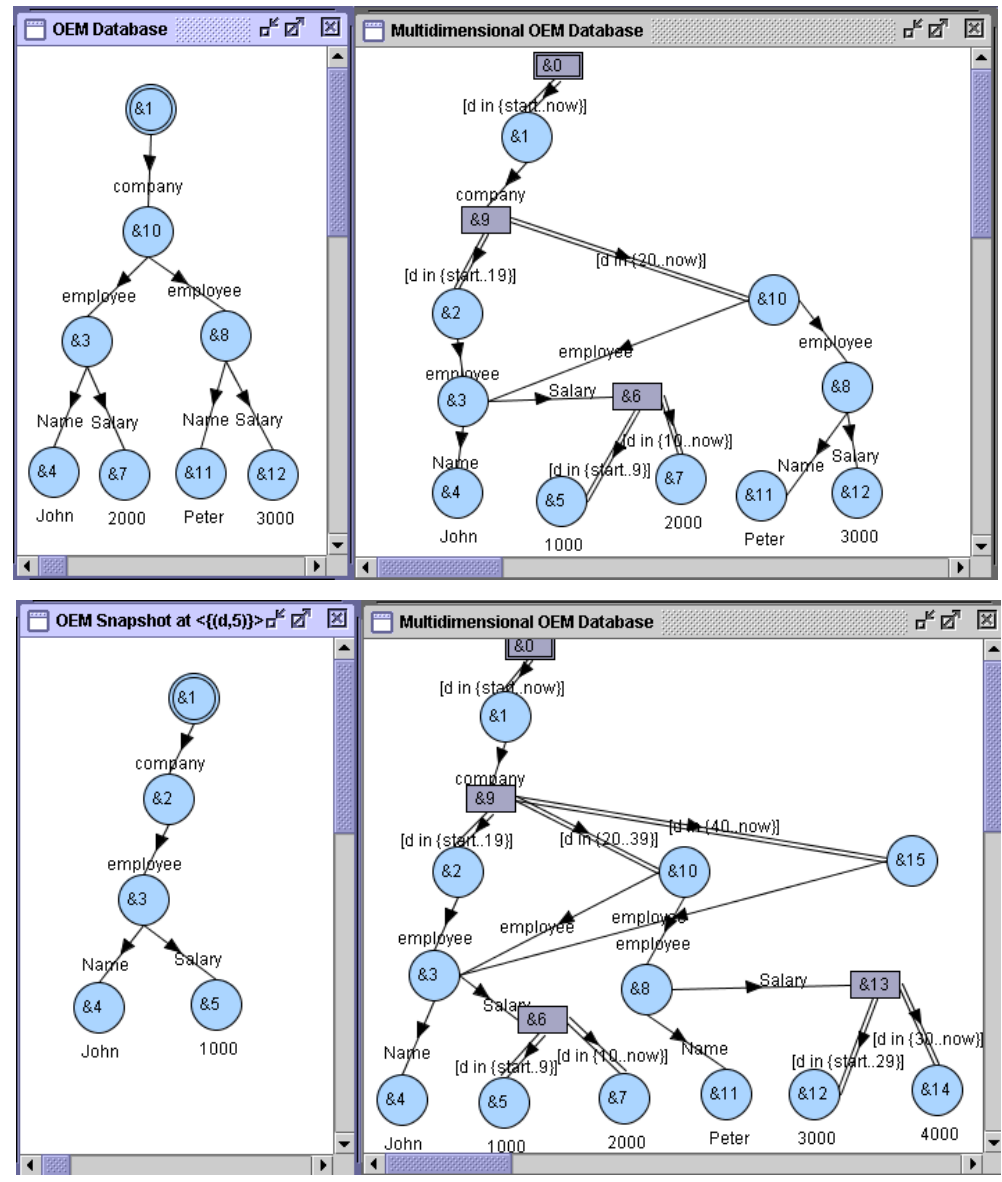

(b)

Fig. 4. Example database after (a) two sequences of basic changes, and (b) four sequences of basic changes upon the initial database state.

Figure 5(a) shows a facet with id $\& 3$ whose value is changed from " $A$ " to " $\mathrm{B}$ " through a call to updateCNode. Figure 5(b) shows the result of an addEEdge operation. Finally, figure 5(c) depicts the addCEdge basic operation. Among MOEM basic operations not shown in Figure 5, remEEdge is very similar to addEEdge; the difference is that an entity edge is removed from facet $\& 8$ instead of being added. In addition, remCEdge is similar to addCEdge: instead of adding one context edge to \&6, one is removed. Finally, the MOEM basic operations createC Node and create $M N$ Node are mapped to themselves; $G^{\prime}$ will record the change when the new nodes are connected to the rest of the graph $G$ through calls to addEEdge or addCEdge.

An MOEM graph $G^{\prime}$ constructed through the process outlined above represents the history of the MOEM graph $G$. In contrast to the case of OEM histories, where a world is defined by only one dimension $d$ representing time, in the case 


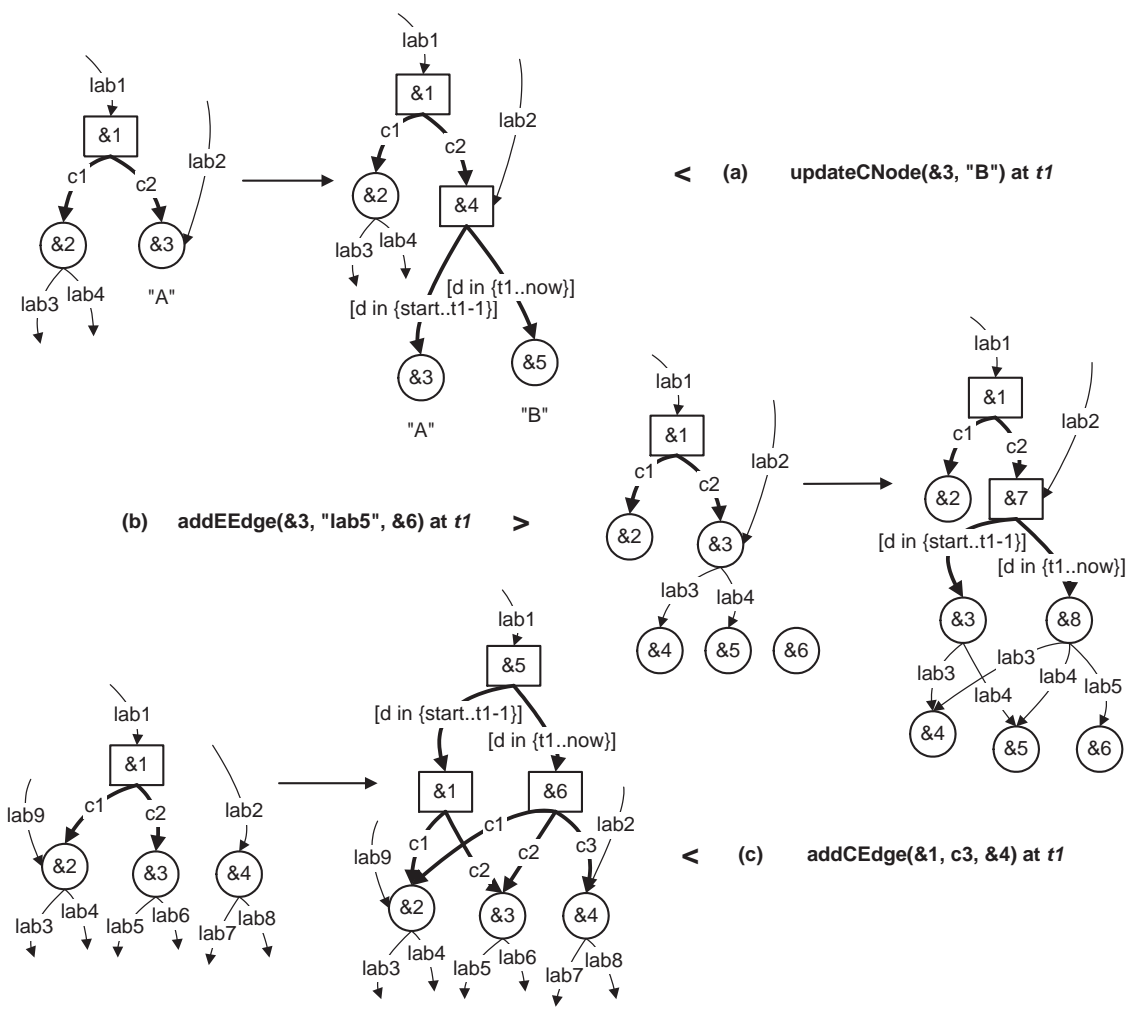

Fig. 5. Modeling Multidimensional OEM basic operations with MOEM.

of MOEM histories a world for $G^{\prime}$ in general involves more than one dimensions, including the time dimension $d$. Therefore, by specifying a value $t$ for $d$ we actually define the set of worlds for which $d=t$. In that set, dimensions other than $d$ may have any combination of values from their respective domains. The process of reducing an MOEM graph under a set of worlds, instead of under a single world, is called partial reduction and, as with full reduction, involves intersecting the given set of worlds with those represented by the inherited contexts of edges and nodes in the graph. Therefore, by applying the process of partial reduction to $G^{\prime}$ for any time instance $t \in T, G^{\prime}$ gives the snapshot of the MOEM database $G$ at that time instance.

\section{Conclusions and Future Work}

In this paper we explained in depth how Multidimensional OEM, a graph model for context-dependent semistructured data, can be used to represent the history of an OEM database. We discussed the MOEM properties in this particular case, and showed that temporal OEM snapshots can be obtained from MOEM. We presented OEM History, an implemented system, and demonstrated through an 
example the process of using an underlying MOEM database to model OEM changes. In addition, we showed that MOEM is capable to model changes occurring not only in conventional OEM databases, but in Multidimensional OEM databases as well.

The applicability of MOEM is not exhausted in representing histories of semistructured data; context-dependent data are of increasing importance in a global environment such as the Web. We have implemented a set of tools for MSSD, which we used to develop the OEM History application. We continue extending this infrastructure that will facilitate the implementation of new MSSD and MOEM applications. Our current work is focused on the implementation of $M Q L$, a multidimensional query language.

\section{References}

1. S. Abiteboul, P. Buneman, and D. Suciu. Data on the Web: From Relations to Semistructured Data and XML. Morgan Kaufmann Publishers, 2000.

2. S. Abiteboul, D. Quass, J. McHugh, J. Widom, and J. L. Wiener. The Lorel Query Language for Semistructured Data. Int. J. on Digital Libraries, 1(1):68-88, 1997.

3. T. Amagasa, M. Yoshikawa, and S. Uemura. A Data Model for Temporal XML Documents. In M. Ibrahim, J. Kung, and N. Revell (eds), Database and Expert Systems Applications (DEXA'00), LNCS 1873, pages 334-344. Springer, 2000.

4. S. S. Chawathe, S. Abiteboul, and J. Widom. Managing Historical Semistructured Data. Theory and Practice of Object Systems, 24(4):1-20, 1999.

5. M. Gergatsoulis, Y. Stavrakas, and D. Karteris. Incorporating Dimensions to XML and DTD. In H. C. Mayr, J. Lanzanski, G. Quirchmayr, and P. Vogel, editors, Database and Expert Systems Applications (DEXA' 01), Proceedings, Lecture Notes in Computer Science, Vol. 2113, pages 646-656. Springer-Verlag, 2001.

6. M. Gergatsoulis, Y. Stavrakas, D. Karteris, A. Mouzaki, and D. Sterpis. A Webbased System for Handling Multidimensional Information through MXML. In A. Kaplinskas and J. Eder, editors, Advances in Databases and Information Systems (ADBIS' 01), LNCS, Vol. 2151, pages 352-365. Springer-Verlag, 2001.

7. T. Mitakos, M. Gergatsoulis, Y. Stavrakas, and E. V. Ioannidis. Representing Timedependent Information in Multidimensional XML. Journal of Computing and Information Technology, 9(3):233-238, 2001.

8. B. Oliboni, E. Quintarelli, and L. Tanca. Temporal Aspects of Semistructured Data. In Proc. of the 8th International Symposium on Temporal Representation and Reasoning (TIME-01), pages 119-127, 2001.

9. Y. Stavrakas and M. Gergatsoulis. Multidimensional Semistructured Data: Representing Context-dependent Information on the Web. In Proc. of the 14 th Int. Conf. on Advanced Information Systems Engineering, Toronto, Canada, May 2002.

10. D. Suciu. An Overview of Semistructured Data. SIGACT News, 29(4):28-38, December 1998. 